\title{
Disturbance Observer-Based Super-Twisting Control for the Inertia Wheel Inverted Pendulum
}

\author{
Afef Hfaiedh \\ (a) University of Carthage, National Engineering School of Carthage \\ (b) Laboratory of Research in Automatic (LA.R.A) \\ Tunis, Tunisia \\ afef.hfaiedh@enicar.u-carthage.tn
}

\author{
Ahmed Chemori \\ LIRMM, University of Montpellier, CNRS \\ Montpellier, France \\ ahmed.chemori@lirmm.fr
}

\author{
Afef Abdelkrim ${ }^{a, b}$ \\ afef.a.abdelkrim@ieee.org
}

\begin{abstract}
In this paper, the problems of stabilization and disturbance rejection in the control of class $I$ of underactuated mechanical systems (UMS) are addressed. Based on a global change of coordinates, the original dynamic model is transformed into a strict-feedback form and a Super-Twisting (STW) controller is designed to resolve the stabilization problem. To deal with the problem of uncertainties and external disturbances in UMS, a disturbance observer (DO) is proposed for the estimation of the input disturbances in the aim of compensating them in the controller and improving the performance and robustness of the resulting closed-loop system. Indeed, the proposed observer-based scheme is compared with the standard STW controller. Both controllers have been implemented and validated through real-time experiments on the inertia wheel inverted pendulum. The obtained results show clearly the superiority of the proposed observer-based STW control scheme and its effectiveness in terms of external disturbance rejection.
\end{abstract}

Index Terms-Underactuated mechanical system, Stabilization, Super-Twisting controller, Disturbance Observer.

\section{INTRODUCTION}

Underactuation in mechanical systems is an attractive case study area of research for control and robotic community. Different challenging control problems and various applications exist in real life. For instance, walking robots, aerospace vehicles, and marine vehicles are some examples of nonlinear applications. Compared to fully actuated systems, the restriction of having fewer control inputs than degrees of freedom complicates the design of the control approach. Besides of their complex nonlinear dynamics and the nonlinear coupling between the directly actuated and the underactuated degrees of freedom, the instability of their internal dynamics leads to a nonminimum phase behaviour. Furthermore, the dynamic model of UMS may include some non-holonomic and non-integrable constraints. Indeed, the level of complexity in the control design depends on the degree of underactuation, thus the linearization and conventional control approaches do not resolve the control problem of high-order underactuated systems. For instance, if the obtained underactuated system after a linearization is uncontrollable, the stabilization may not be achieved by a smooth state feedback controller. Sliding mode control (SMC) was one of the most promising controllers studied for nonlinear systems [1] and commonly designed in various UMSs due to its robustness and insensitivity to parametric uncertainties. Xu and Ozguner [2] proposed a sliding-mode controller for a special class of UMSs transformed into a cascaded representation where the proposed control approach was justified by simulation on the TORA and inverted pendulum systems. After a transformation in a feedforward form, a SMC was designed in [3] for the slosh-container system which belongs to the second class of UMSs according to the proposed classification in [4]. In [5] the authors proposed a nonlinear disturbance observer-based SMC approach for uncertain rotational pendulum system. Another SMC approach based on the LMI method is proposed to control a complex Tethered Satellite [6]. Other extensions of SMC based on fuzzy logic methods were also designed in the literature for UMSs in [7] [8] [9] [10].

The main disadvantage of the first SMC especially in real-time implementation is the high-frequency oscillatory phenomenon [11] which affects the performances of the system and increase the energy consumption. As UMSs have fewer actuators than degrees of freedom [12], they are more sensitive to variation in the control input signal than fully actuated systems. Thus the chattering phenomenon is not convenient for such systems since it can lead to low control accuracy, the instability of the system and permanently damage of the actuators. In order to counteract this limitation which is related to the discontinuous terms in the control law, the use of a smooth approximation can resolve the problem. However, this solution is not always promising for all complex nonlinear mechanical systems. Furthermore, the invariance property of the sliding mode control will be lost. To attenuate the chattering phenomenon [13], other effective solutions are proposed based on genetic algorithms; however, these control methods require a careful selection of parameters and a large execution 
time. Higher-Order Sliding Mode Control (HOSMC) based strategies are also developed in the literature to overcome the above-cited problems and to deal with the control problems related to UMSs [14] [15] [16].

Among the most effective second-order sliding mode approaches, Super-Twisting Control has been widely implemented by researchers, is a second-order SMC, designed to drive both the sliding variable and its derivative to zero in finite time. It has the main properties of firstorder sliding mode controller with a continuous control law since the discontinuous term inducing the switching function is integrated into the control law expression [17]. It has the benefits of compensating Lipschitz uncertainties [18] and attenuating the chattering without eliminating it [19]; however, it requires the knowledge of the disturbance boundaries which is difficult to be practically estimated. In this paper, we propose to improve the robustness of the STW controller by including some knowledge of the input external disturbances in the considered class I of underactuated systems. To address the problem of underactuation, the system is transformed in a strict-feedback form. This conversion represents the system into cascade interconnection of a linear subsystem and a nonlinear core subsystem, which makes the control problem more simplified. To improve the disturbances rejection of the system, a classical disturbance observer is proposed to estimate the external input disturbances. The effectiveness of the proposed disturbance observerbased STW control is evaluated through its experimental implementation to stabilize the inertia wheel inverted pendulum (IWIP) which has commonly served as a benchmark testbed in many research works [20] [21] [22] [23].

The rest of the paper is outlined as follows. In Section II we present a general background on the transformation of this class in strict-feedback form. In section III, the dynamic model of the IWIP and an illustration of the transformation are presented. The proposed STW controller and the disturbance observer are illustrated in section IV. Experimental results with a comparative analysis are displayed in Section V. In the end, conclusion and future works are presented in section VI.

\section{Class I OF UNDERACTUATED MEChANiCAL SYSTEMS}

Most of real-life UMSs are nonlinear high-order systems. Due to their different properties, no common and general control scheme is appropriate for all UMSs. The main idea is to refer to the classification proposed in [4] and to use an explicit change of coordinates which uncouple the original system into a cascade interconnection of a simple reduced nonlinear and linear subsystems. The main motivation behind this transformation is the representation of the system model into other simplified structure and to makes the control analysis more sim- plified. The general expression of a dynamical model of second-order UMS can be represented by

$$
M(q) \ddot{q}+C(q, \dot{q}) \dot{q}+G(q)=F(q) \tau
$$

where $\tau=\left[\tau_{1}, \tau_{2}\right]^{T} \in \mathbb{R}^{m}$ is the vector of control input, $M(q)$ and $C(q)$ denote the inertia matrix and the matrix of Coriolis and Centrifugal respectively, $G(q)$ and $F(q)=\left[0, I_{m}\right]^{T} \in \mathbb{R}^{n \times m} m<n$ represent respectively the gravity vector and the non-square matrix of external forces. The vector of generalized coordinates is $q=\left(q_{1}, q_{2}\right) \in \mathbb{R}^{n-m} \times \mathbb{R}^{m}$ where the first coordinate $q_{1}$ is a non-actuated configuration and the second coordinate $q_{2}$ is actuated.

Considering the dynamical model of system (1), with the lack of control input in the first equation

$\left[\begin{array}{ll}m_{11}(q) & m_{12}(q) \\ m_{21}(q) & m_{22}(q)\end{array}\right]\left[\begin{array}{l}\ddot{q}_{1} \\ \ddot{q}_{2}\end{array}\right]+\left[\begin{array}{l}h_{1}(q, \dot{q}) \\ h_{2}(q, \dot{q})\end{array}\right]=\left[\begin{array}{c}0 \\ \tau\end{array}\right]$

where $m_{11}(q), m_{12}(q), m_{21}(q), m_{22}(q)$ represent the components of the inertia matrix and $h_{1}(q, \dot{q}), h_{2}(q, \dot{q})$ contain the Coriolis, Centrifugal and gravity terms.

If the following assumption is verified, the system (2) with two degrees of freedom and only one control input can be represented into strict-feedback form.

Assumption1: Considering the vector of generalized coordinates $q=\left(q_{1}, q_{2}\right)$, a global change of coordinates can be defined as follows if the term $m_{11}^{-1}\left(q_{2}\right) m_{12}\left(q_{2}\right)$ is integrable.

$$
\begin{aligned}
& z_{1}=q_{1}+\gamma\left(q_{2}\right) \\
& z_{2}=m_{11}\left(q_{2}\right) p_{1}+m_{12}\left(q_{2}\right) p_{2} \\
& \xi_{1}=q_{2} \\
& \xi_{2}=p_{2}
\end{aligned}
$$

where $\gamma\left(q_{2}\right)=\int_{0}^{q_{2}} m_{11}^{(-1)}(\theta) m_{12}(\theta) d \theta \quad$ and $\left[z_{1}, z_{2}, \xi_{1}, \xi_{2}\right]^{T}$ represent the new state vector. Using the explicit change of coordinates, we obtain a new representation of the system in strict-feedback form described as follows

$$
\begin{aligned}
& \dot{z}_{1}=m_{11}^{-1}\left(\xi_{1}\right) z_{2} \\
& \dot{z}_{2}=g_{1}\left(z_{1}-\gamma\left(\xi_{1}\right), \xi_{1}\right) \\
& \dot{\xi}_{1}=\xi_{2} \\
& \dot{\xi}_{2}=u
\end{aligned}
$$

where $g_{1}\left(q_{1}, q_{2}\right)=-\frac{\partial V(q)}{\partial q_{1}}, V(q)$ denotes the potential energy of the system and $u$ is the new control input from collocated partial feedback linearization. The torque and the new control terms are related through these expressions

$$
\begin{aligned}
\tau & =\alpha(q) u+\beta(q, \dot{q}) \\
\alpha(q) & =m_{22}(q)-m_{21}(q) m_{11}^{-1}(q) m_{12}(q) \\
\beta(q, \dot{q}) & =h_{2}(q, \dot{q})-m_{21} m_{11}^{-1}(q) h_{1}(q, \dot{q})
\end{aligned}
$$

In the next section, the strict-feedback simplified model is adopted for the representation of the inertia wheel pendulum system. 


\section{THE INERTIA WHEEL INVERTED PENDULUM}

The inertia wheel inverted pendulum presented in Fig. 1 was widely considered as an academic system to implement and study new approaches for the stabilization control problem [20] [24] [25] or generation of stable periodic limit cycles [26] [27] [28] [29]. It has two degrees of freedom and one control input. The pendulum angle $\theta_{1}$ according to the vertical is unactuated and the angle between the body and the inertia wheel $\theta_{2}$ is actuated. To stabilize the system, the design of the control input is required to act on the inertia wheel and to maintain the unactuated pendulum in its unstable angular equilibrium position. According to the Euler-Lagrange

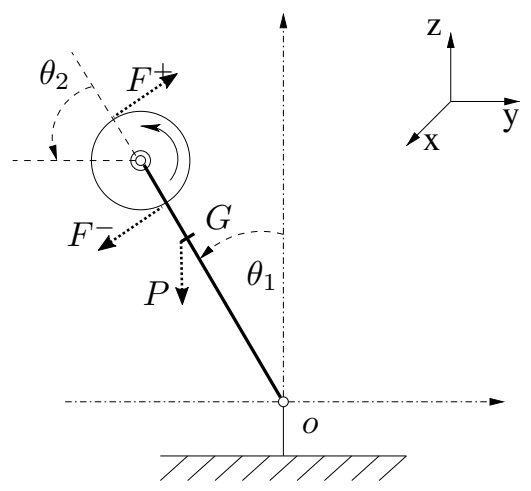

Fig. 1. Schematic view of the system: the first joint $\theta_{1}$ is unactuated, while the second $\theta_{2}$ is actuated.

method, the equation of motion of the system is obtained

$$
L=\frac{1}{2}\left(I_{1} \dot{\theta}_{1}^{2}+i_{2}\left(\dot{\theta}_{1}+\dot{\theta}_{2}\right)^{2}\right)-m_{0} g \cos \left(\theta_{1}\right)
$$

The dynamic model of the IWIP [30] [22] is presented as follows

$$
\left[\begin{array}{ll}
I+i_{2} & i_{2} \\
i_{2} & i_{2}
\end{array}\right]\left[\begin{array}{l}
\ddot{\theta}_{1} \\
\ddot{\theta}_{2}
\end{array}\right]-\left[\begin{array}{l}
m_{0} g \sin \left(\theta_{1}\right) \\
0
\end{array}\right]=\left[\begin{array}{l}
0 \\
\tau
\end{array}\right]
$$

where $\theta=\left[\theta_{1}, \theta_{2}\right]$, represents the vector of generalized coordinates, $\tau$ is the torque applied on the wheel. The two constants $I$ and $m_{0}$ are expressed by $I=m l^{2}+M L^{2}+i_{1}$ and $m_{0}=m l+M L$. The dynamic parameters of the IWIP are presented in TABLE I.

TABLE I

SUMMARY OF THE DYNAMIC PARAMETERS OF THE IWIP

\begin{tabular}{|c|c|c|}
\hline Parameter & Description & Value \\
\hline$i_{1}$ & Inertia of the pendulum body & $0.031468\left[\mathrm{~kg} \cdot \mathrm{m}^{2}\right]$ \\
$i_{2}$ & Inertia of the Wheel & $4.17610^{-4}\left[\mathrm{~kg} \cdot \mathrm{m}^{2}\right]$ \\
$l$ & Body center of mass position & $0.06[\mathrm{~m}]$ \\
$L$ & Wheel center of mass position & $0.044[\mathrm{~m}]$ \\
$m$ & Body mass & $3.228[\mathrm{~kg}]$ \\
$M$ & Wheel mass & $0.86422[\mathrm{~kg}]$ \\
$g$ & Constant of gravitational acceleration & $9.8\left[\mathrm{~m} \cdot \mathrm{s}^{-2}\right]$ \\
\hline
\end{tabular}

\section{A. Strict-feedback form of the system}

The inertia matrix of the system is constant and the expression $m_{11}^{-1}\left(q_{2}\right) m_{12}\left(q_{2}\right)$ is integrable. The model (12) of the system verifies the Assumption 1. Therefore, the resulting strict-feedback system can be obtained through this new configuration.

$$
\begin{gathered}
z_{1}=\frac{\partial L}{\partial \dot{\theta}_{1}}=\left(I_{1}+i_{2}\right) \dot{\theta}_{1}+i_{2} \dot{\theta}_{2} \\
z_{2}=\theta_{1} \\
z_{3}=\dot{\theta}_{2}
\end{gathered}
$$

where $Z=\left[z_{1}, z_{2}, z_{3}\right]$ represents the new system states of the transformed system, $\theta_{1}, \dot{\theta}_{1}, \dot{\theta}_{2}$ are respectively the original angular position, the angular velocity of the pendulum and the angular velocity of the wheel. Using the new coordinates in (13) (14) and (15) the dynamics is transformed into a strict-feedback form as follows

$$
\begin{gathered}
\dot{z}_{1}=\frac{\partial L}{\partial \theta_{1}}=m_{0} g \sin \left(z_{2}\right) \\
\dot{z}_{2}=\frac{z_{1}}{I_{1}+i_{2}}-\frac{i_{2} z_{3}}{I_{1}+i_{2}} \\
\dot{z}_{3}=u
\end{gathered}
$$

The obtained system is a cascade connection between a reduced nonlinear subsystem and linear subsystem where the new coordinate $z_{2}$ is considered as a virtual control input of the $z_{1}$-subsystem described in (16). The stability of this latter can be verified via Lyapunov stability analysis. Let a Lyapunov function expressed as.

$$
V\left(z_{1}\right)=\frac{z_{1}^{2}}{2}
$$

then the derivative of the Lyapunov function (19) is expressed

$$
\dot{V}\left(z_{1}\right)=z_{1} \dot{z}_{1}
$$

If we consider a sigmoidal desired trajectory for the $z_{1}$ subsystem and its first derivative expressed by

$$
\begin{gathered}
\theta_{1 d}=z_{2 d}=-\arctan \left(z_{1}\right) \\
\dot{\theta}_{1 d}=\frac{m_{0} g \sin \left(z_{2}\right)}{\left(1+\left(z_{1}^{2}\right)\right)}
\end{gathered}
$$

we substitute (16) into (20), then replacing (21) in the expression, we obtain .

$$
\begin{aligned}
& \dot{V}\left(z_{1}\right)=z_{1} m_{0} g \sin \left(z_{2}\right) \\
& \dot{V}\left(z_{1}\right)=z_{1} m_{0} g \sin \left(-\arctan \left(z_{1}\right)\right) \\
& \dot{V}\left(z_{1}\right)=-m_{0} g z_{1} \frac{z_{1}}{\sqrt{1+z_{1}^{2}}}
\end{aligned}
$$

if $z_{1}>0$, then, $\dot{V}\left(z_{1}\right)<0$

if $z_{1}=0$, then, $\dot{V}\left(z_{1}\right)=0$

if $z_{1}<0$, then, $\dot{V}\left(z_{1}\right)<0$

Therefore by the valid Lyapunov function (19) and the analysis of three cases of $z_{1}$, the convergence of the system is verified. 


\section{PROPOSED CONTROL APPROACH}

\section{A. Design of the Super-Twisting Controller}

The super-twisting sliding mode control is widely used in nonlinear control systems due to its effectiveness to solve the problem of the chattering phenomenon and to hide the high-frequency oscillation. Furthermore, it does not require information about the boundaries of the disturbance and any information concerning the derivative of the sliding variable. The proposed continuous STW control law is described as

$$
\begin{aligned}
u & =-k_{1}|\sigma|^{\frac{1}{2}} \operatorname{sign}(\sigma)+w \\
\dot{w} & =-k_{2} \operatorname{sign}(\sigma)
\end{aligned}
$$

where $\sigma$ is the sliding variable, sign denotes the standard signum function, $k_{1}>0$ and $k_{2}>0$ are positive constant controller's gains. It is worth to note, that the proposed control law (26) cannot stabilize the system if it is applied directly to the original model (12). Indeed, the stabilization requires the transformation of the system in the strict-feedback form to define the sliding variable with respect to the desired trajectory expressed in (21). The final control law term is expressed in (8). The proposed sliding variable required to implement the STW controller in our case is expressed as

$$
\sigma=\left(\theta_{1 d}-\theta_{1}\right)+\alpha_{1}\left(\dot{\theta}_{1 d}-\dot{\theta}_{1}\right)
$$

where $\theta_{1 d}$ and $\dot{\theta}_{1 d}$ are the proposed desired trajectory and its first derivative respectively, $\alpha_{1}$ is a positive constant to be selected. In the next section, we propose the use of a linear disturbance observer (DO) to deal with the external disturbance rejection of this class of secondorder underactuated mechanical systems.

\section{B. Disturbance Observer}

Uncertainties and external disturbances are extremely inevitable in UMSs. Due to the lack of actuator and the nonlinear coupling between the coordinates, the system become more sensitive to uncertainties and small external disturbances can conduct to the destabilization and instability of the system. On this basis, we propose the use of disturbance observer which is widely employed as a tool for disturbance rejection especially in the practice environment. The proposed DO can be easily implemented in real-time applications to improve the robustness of the closed-loop system, to compensate the system uncertainties and to reject the input disturbances. In our case, we deal with a second-order system, it is reasonable to use the basic form of a disturbance observer. Assuming that the model of the IWP can be represented by

$$
M(q) \ddot{q}+H(q, \dot{q})=\tau+d
$$

where $q \in \mathbb{R}^{2}, \dot{q} \in \mathbb{R}^{2}, \tau$ is the control input and $d=$ $\left[d_{1}, d_{2}\right]^{T} \in \mathbb{R}^{2}$ is a vector of disturbance torque which can be the friction, unmodeled dynamics in independent joint control or external disturbing torque. The basic idea is to use the system states and input torque as input and then to estimate all the unknown external torque imposed on the system. The expression (29) can be written as

$$
d=M(q) \ddot{q}+H(q, \dot{q})-\tau
$$

The expression of the proposed conventional observer is described as follow

$$
\dot{\hat{d}}=-L_{1}(q, \dot{q}) \hat{d}+L_{1}((q, \dot{q}))(M(q) \ddot{q}+H(q, \dot{q})-\tau)
$$

where $L_{1}(q, \dot{q})$ is a diagonal matrix. $d$ is considered to be an unknown disturbance torque and the measurement of the velocity and acceleration is provided by numerical derivation. The observer error is expressed by

$$
e=d-\hat{d}
$$

Assuming that the disturbance is constant during the sampling period and there is no prior information about its derivative, we consider that $(\dot{d}=0)$, the resulting observer error system can be written by

$$
\dot{e}+L_{1}(q, \dot{q}) e=0
$$

The system (33) has the form of a linear state space model $\dot{X}=A X$ where $A=-L_{1}$. If all real parts of the eigenvalues of $A$ are negative, the system is asymptotically stable. The global asymptotic stability of (33) can be verified by the choice of $L_{1}(q, \dot{q})=\operatorname{diag}(\varphi, \varphi)$ is a diagonal constant positive matrix.

\section{EXPERIMENTAL RESULTS}

In this section, we will include some experimental results to validate the performance of the proposed approach.

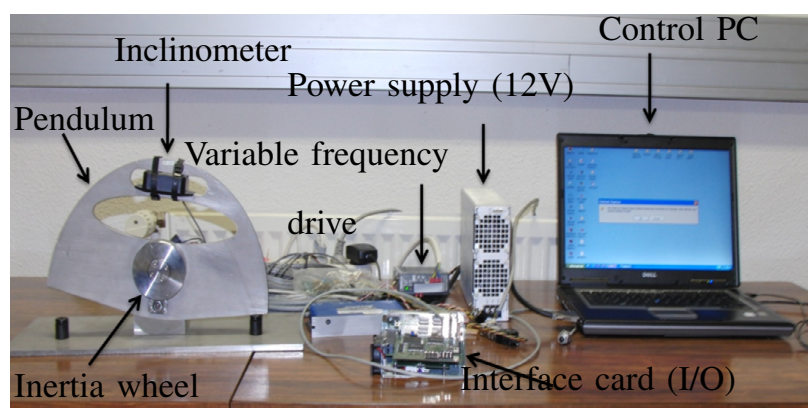

Fig. 2. View of the IWIP experimental testbed

The experiment was conducted on the benchmark of the inertia wheel inverted pendulum [24] [25] [31] as shown in Fig. 2. For comparison purposes, as a first step, we implement only the STW controller then the proposed STW controller combined with a basic disturbance observer. To compare the effectiveness of both control schema against disturbance, two punctual disturbances were applied to the pendulum body. For the standard STW controller, the external disturbances were applied at time $t=10 \mathrm{~s}$ and $t=20.5 \mathrm{~s}$. For the proposed scenario, the disturbances were applied by pushing the pendulum 

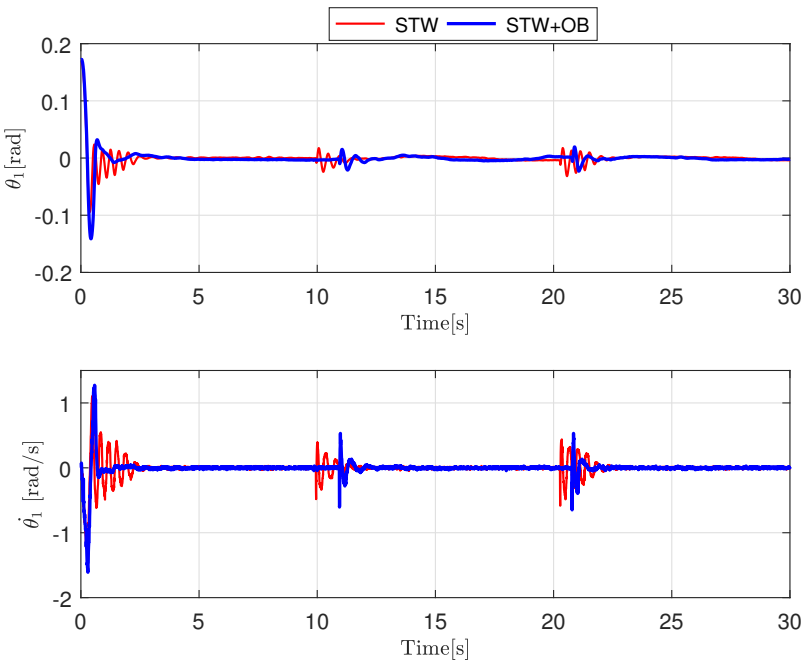

Fig. 3. Obtained experimental results (top): Pendulum angular position versus time, (bottom): pendulum angular velocity versus time.
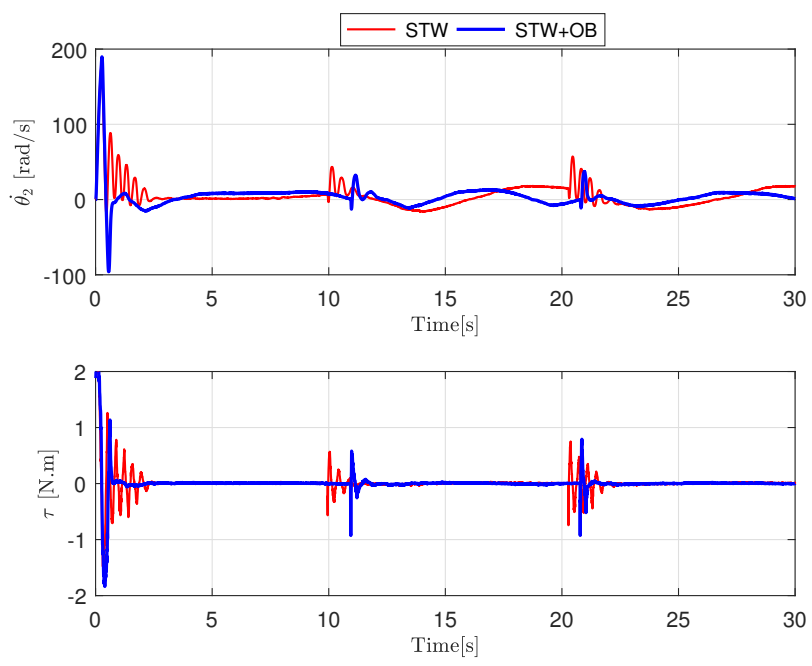

Fig. 4. Obtained experimental results (top): velocity of the inertia wheel versus time, (bottom) the control input (torque) versus time.

at $t=10.5 \mathrm{~s}$ and $t=21 \mathrm{~s}$. The considered scenario was achieved in the best possible way in order to apply the same disturbance at approximately the same time. The parameters of the real benchmark are summarized in TABLE I. The parameters used for the control scheme are $K_{1}=1.2, K_{2}=0.15, \alpha_{1}=0.1, L_{1}=\operatorname{diag}(5,5)$. The evolution of the system states and the control input are depicted in Fig. 3.

We can easily conclude through the evolution of pendulum position Fig. 3.(b) and the angular velocities of the pendulum Fig. 3.(b) and the inertia wheel versus time Fig. 4.(a), that the convergence of the system state has been established for both control schema and they are able to compensate the disturbances. However, more oscillations are shown in case of the STW controller and we can observe a faster convergence and better external disturbance rejection of the proposed approach than the
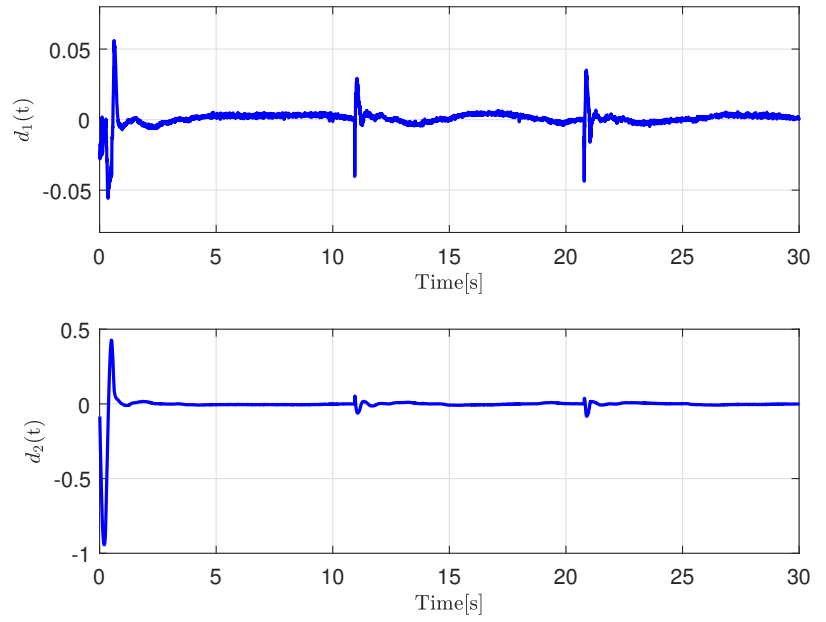

Fig. 5. Obtained experimental results (top): Evolution of the estimated disturbance $d_{1}(t)$ versus time, (bottom): Evolution of the estimated disturbance $d_{2}(t)$ versus time

standard STW controller. The evolution of the control input for both control scheme is depicted in Fig. 4.(b). The control input signal of the proposed approach is more smoother with respect to the control input of the standard STW approach. The evolution of the estimated disturbances is illustrated in Fig. 5, we can see the effect of the punctual disturbances applied in the pendulum body by the two peaks in the evolution of the estimated disturbances.

\section{CONCLUSION AND FUTURE WORK}

In this paper, a disturbance observer based STW control is proposed for the stabilization of the inertia wheel inverted pendulum which belongs to the class I of UMSs. Real-time experiments show the performance and the effectiveness of the proposed control scheme and its robustness towards external punctual disturbances. Our future work will be focused on the extension of this control approach for more complex and high-order underactuated mechanical systems.

\section{REFERENCES}

[1] V. Utkin, Sliding Modes in Control and Optimization. SpringerVerlag Berlin Heidelberg, 1992.

[2] R. Xu and U. Özgüner, "Sliding mode control of a class of underactuated systems," Automatica, vol. 44, pp. 233-241, 01 2008.

[3] P. S. Thakar, B. Bandyopadhyay, and P. S. Gandhi, "Sliding mode control for a class of underactuated systems using feedforward normal form: A slosh-container system," in 2014 13th International Workshop on Variable Structure Systems (VSS), Nantes, France, 2014, pp. 1-6.

[4] R. Olfati-Saber, "Nonlinear control of underactuated mechanical systems with application to robotics and aerospace vehicles," Ph.D. dissertation, Massachusetts Institute of Technology, Cambridge, MA, USA, 2001.

[5] K. Majumder and B. M. Patre, "Sliding mode control for underactuated mechanical systems via nonlinear disturbance observer : stabilization of the rotational pendulum," Int. J. Dynam. Control, vol. 6, no. 6, p. 1663-1672, 2018. 
[6] N. Keshtkar, S. Keshtkar, J. A. Moreno, A. Poznyak, and H. Kojima, "Lmi-based sliding mode control of an underactuated control moment gyroscope system $* *$ the authors thank the grant sep-conacyt number 251552 and dgapa-unam for the financial support." IFAC-PapersOnLine, vol. 51, no. 13, pp. 291 - 296 , 2018, 2nd IFAC Conference on Modelling, Identification and Control of Nonlinear Systems MICNON 2018.

[7] S. Li, D-T. Liu, and P-J. Wang, "Fuzzy sliding mode control for a class of underactuated systems," in 2013 International Conference on Machine Learning and Cybernetics, vol. 01, Tianjin, China, 2013, pp. 178-183.

[8] S. Mahjoub, F. Mnif, and N. Derbel, "Radial-basis-functions neural network sliding mode control for underactuated manipulators," in 10th International Multi-Conferences on Systems, Signals Devices 2013 (SSD13), Hammamet, Tunisia, 2013, pp. 1-6.

[9] R. Hernández and F. Jurado, "Adaptive fuzzy sliding mode control of an inverted pendulum mounted on a ball system," in 2018 IEEE Latin American Conference on Computational Intelligence (LACCI), Gudalajara, Mexico, 2018, pp. 1-6.

[10] X. Huang, A. L. Ralescu, H. Gao, and J. Wen, "Adaptive hierarchical sliding mode control based on fuzzy neural network for an underactuated system," in 2018 IEEE International Conference on Fuzzy Systems (FUZZ-IEEE), Rio de Janeiro, Brazil, 2018, pp. $1-7$.

[11] G. Bartolini, A. Ferrara, and E. Usai, "Chattering avoidance by second-order sliding mode control," IEEE Transactions on Automatic Control, vol. 43, no. 2, pp. 241-246, Feb 1998.

[12] J. Ghommam and A. Chemori, "Adaptive rbfnn finite-time control of normal forms for underactuated mechanical systems," Nonlinear Dyn, vol. 90, no. 1, p. 301-315, 2017.

[13] V. Utkin and H. Lee, "Chattering problem in sliding mode control systems," in International Workshop on Variable Structure Systems VSS'06., Sardinia, Italy, 2006, pp. 346-350.

[14] S. Riachy, Y. Orlov, T. Floquet, R. Santiesteban, and J.-P. Richard, "Second-order sliding mode control of underactuated mechanical systems i: Local stabilization with application to an inverted pendulum," International Journal of Robust and Nonlinear Control, vol. 18, pp. 529-543, 2008.

[15] R. Santiesteban, T. Floquet, Y. Orlov, S. Riachy, and J.-P. Richard, "Second-order sliding mode control of underactuated mechanical systems II: Orbital stabilization of an inverted pendulum with application to swing up/balancing control," International Journal of Robust and Nonlinear Control, vol. 18, pp. 544-556, 2008.

[16] S. Mahjoub, F. Mnif, and N. Derbel, "Second-order sliding mode approaches for the control of a class of underactuated systems," International Journal of Automation and Computing, vol. 12, p. 134-141, 2015.

[17] L. F. Y. Shtessel, C. Edwards and A. Levant, Sliding Mode Control and Observation, ser. Control Engineering. Springer New York, 2014, vol. Intuitive Theory of Sliding Mode Control.

[18] A. Chalanga, S. Kamal, L. M. Fridman, B. Bandyopadhyay, and J. A. Moreno, "Implementation of super-twisting control: Super-twisting and higher order sliding-mode observer-based approaches," IEEE Transactions on Industrial Electronics, vol. 63, no. 6, pp. 3677-3685, 2016.

[19] Y. Shtessel, F. Plestan, and M. Taleb, "Lyapunov design of adaptive super-twisting controller applied to a pneumatic actuator," IFAC Proceedings Volumes, vol. 44, no. 1, pp. 3051 - 3056, 2011 , 18th IFAC World Congress.

[20] N. Khraief Haddad, A. Chemori, and S. Belghith, "Robustness enhancement of ida-pbc controller in stabilising the inertia wheel inverted pendulum: theory and real-time experiments," International Journal of Control, vol. 91, no. 12, pp. 2657-2672, 2018.

[21] S. Andary, A. Chemori, and S. Krut, "Control of the underactuated inertia wheel inverted pendulum for stable limit cycle generation," Advanced Robotics, vol. 23, no. 15, pp. 1999-2014, 2009.

[22] S. Andary, A. Chemori, and S. Krut, "Estimation-based disturbance rejection in control for limit cycle generation on inertia wheel inverted pendulum testbed," in 2009 IEEE/RSJ International Conference on Intelligent Robots and Systems, St Louis, USA, 2009, pp. 1302-1307.

[23] A. Hfaiedh, A. Chemori, and A. Abdelkrim, "Rise controller for class I underactuated mechanical systems: Design and real-time experiments," in The 3rd International Conference on Electromechanical Engineering (ICEE'2018), Skikda, Algeria, 2018.
[24] N. Khraief Haddad, A. Chemori, and S. Belghith, "External disturbance rejection in ida-pbc controller for underactuated mechanical systems: From theory to real time experiments," in 2014 IEEE Conference on Control Applications (CCA), Juan Les Antibes, France, Juan Les Antibes, France, Oct 2014, pp. 17471752.

[25] N. Khraief Haddad, A. Chemori, J. J. Pena, and S. Belghith, "Stabilization of inertia wheel inverted pendulum by model reference adaptive ida-pbc: From simulation to real-time experiments," in 2015 3rd International Conference on Control, Engineering Information Technology (CEIT), Tlemcen, Algeria, Tlemcen, Algeria, May 2015, pp. 1-6.

[26] S. Andary, A. Chemori, and S. Krut, "Stable limit cycle generation for underactuated mechanical systems, application: Inertia wheel inverted pendulum," in 2008 IEEE/RSJ International Conference on Intelligent Robots and Systems, Nice, France, 2008, pp. 526-531.

[27] H. Gritli, N. Khraief, A. Chemori, and S. Belghith, "Selfgenerated limit cycle tracking of the underactuated inertia wheel inverted pendulum under ida-pbc," Nonlinear Dynamics, vol. 29, no. 3, 052017.

[28] N. Haddad, S. Belghith, H. Gritli, and A. Chemori, "From hopf bifurcation to limit cycles control in underactuated mechanical systems," International Journal of Bifurcation and Chaos, vol. 27, no. 7, p. 1750104, 062017.

[29] S. Andary and A. Chemori, "A dual model-free control of nonminimum phase systems for generation of stable limit cycles," in 2011 50th IEEE Conference on Decision and Control and European Control Conference, Orlando, FL, USA, 2011, pp. 1387-1392.

[30] C. Zayane-Aissa, T. Laleg-Kirati, and A. Chemori, "Control of a perturbed under-actuated mechanical system," in 2015 IEEE Conference on Control Applications (CCA), Sydney, Australia, 2015, pp. 294-299.

[31] S. Andary, A. Chemori, M. Benoit, and J. Sallantin, "A dual model-free control of underactuated mechanical systems, application to the inertia wheel inverted pendulum," in 2012 American Control Conference (ACC), Montreal, QC, Canada, 2012. 